\title{
Efficient Reciprocating Internal Combustion Engine Operating Under a Regenerative Isobaric Expansion Based Cycle
}

\author{
R. Ferreiro-García ${ }^{1}$, R. Borrás-Formoso ${ }^{1}$, J. Carbia-Carril $^{2}$ \\ ${ }^{1}$ Ind. Eng. Dept \\ E.T.S.N.y M., A Coruña University \\ Campus of Riazor, 15011 A Coruña (Spain) \\ Phone/Fax number:+0034 981 167000,e-mail: ferreiro@udc.es; ramon.borras@udc.es; \\ ${ }^{2}$ Energy and Propulsion Dept. \\ E.T.S.N.y M., A Coruña University \\ Campus of Riazor , 15011 A Coruña (Spain) \\ Phone/Fax number:+0034 981 167000, e-mail: Carbia@udc.es;
}

\begin{abstract}
The work deals with a novel regenerative trilateral internal combustion engine cycle (RTICC) which differs from the conventional Carnot derived thermal gas cycles in that energy absorption and conversion into mechanical work is performed in a single process between two state points of the cycle under an operating pressure which balances the reaction load, so that for a constant load this path function is isobaric. While thermal energy is being absorbed along the expansion process, internal energy, enthalpy, specific volume and entropy increases as mechanical work is delivered.

The analysis of the cycle is presented and results are compared with Carnot factor which conventionally has been considered as a benchmark for all thermal engine cycles. The results show that a relevant advantage of the proposed cycle deals with performance enhancement into a wide range of combustion temperatures. The successful results associated with a compact and simple engine structure, and economically viable, promises a new generation of efficient internal combustion engines.
\end{abstract}

\section{Key words}

Carnot factor, Heat regeneration, Internal combustion engine, Internal energy, Thermal efficiency, Trilateral thermal cycle

\section{Background on conventional thermal cycles}

Conventional thermal cycles so far which are derived from the Carnot cycle have a common characteristic consisting of the processes of heat absorption and energy conversion into mechanical work by means of two different processes, while in the proposed trilateral cycle the transformation due to energy absorption and mechanical work conversion consists of a unique process.

The proposed RTICC Fig.1, consists of three transformations implemented as (1-2) air compression, (2-3) expansion associated to internal combustion and conversion to mechanical work, and (3-1) exhausting flue gas with high grade heat associated to heat regeneration transferring heat to the compressed air.

Some studies regarding trilateral thermal cycles have recently appeared in scientific literature. Thus, for instance, a comparison of trilateral cycles and organic Rankine cycles has been carried out by [1] Johann Fischer (2012) which is a clear contribution to the thermal efficiency enhancement has been reported. Nevertheless, in the proposed present work, a different perspective of trilateral thermal cycles is being considered which are depicted in the Fig.1: the isobaric expansion based trilateral thermal cycles under condensing or noncondensing modes, which also can be regenerative or not as described in [2], which are based on a completely different concept in comparison with the conventional Carnot based thermal cycles, structurally but also in terms of Carnot factor limitations.

Because the technologies applied to the conventional thermal cycles (Diesel and Otto) are mature technologies, they didn't experiment significant advances in recent decades. Thus, some of the most relevant contributions are due to [3] Yanlin Ge et.al., who contributed to the performance of an air-standard Otto cycle with heat transfer loss and variable specific heats of working fluid by using finite-time thermodynamics, [2] Yingru Zhao and Jincan Chen, developed an irreversible dual heat engine model, which can include the Otto and Diesel cycles, used to investigate the influence of the multiirreversibilities mainly resulting from the adiabatic processes, finite time processes and heat leak loss through the cylinder wall on the performance of the cycle, [4] Shaojun Xia, investigated a Diesel cycle engine with internal and external irreversibilities of finite combustion rate of the fuel, friction and heat leakage.

The objective of the study is to efficiently convert thermal energy into mechanical work by means of an internal combustion engine which obeys to a regenerative trilateral 
thermal cycle very different from the trilateral cycle described by [1] Johann Fisher (2012). The proposed conversion method is based on a different thermodynamic cycle: the proposed trilateral thermal cycle (RTICC) is not based on the quadrilateral Carnot cycle but on the trilateral cycle which absorbs energy and develops mechanical work simultaneously along a unique transformation between state points (2)-(3) of the cycle as shown in Fig.1. As a consequence of this contribution, this cycle is not restricted by the Carnot factor constraints. Its maximum thermal efficiency is influenced not only by the ratio of the top to bottom temperatures like for instance in the cycles' analysis described by [5] S.C. Kaushik, V.SivaRedd, S.K.Tyagi (2011), and [6], Jing Li, Gang Pei, Yunzhu Li, Dongyue Wang, Jie Ji (2012) but also by some factors such as the characteristics of the working fluids and regeneration capabilities when regeneration is considered.

The research work deals with a new contribution regarding a trilateral thermal cycle applied on the trilateral regenerative internal combustion engine, so that the work has been organized in 5 sections, where section 2 is devoted to the description of the new concepts included in the proposed contribution, the section 3 describes de analysis of the RTICC engine, in section 4 a case study is included, in which it is proposed the realizable structure of the projected engine. The performance analysis and discussion of results is proposed in a subsection, and finally in section 5 some relevant conclusions are highlighted.

\section{Proposed contribution}

The objective of the study is to convert efficiently thermal energy from fossil fuel combustion to mechanical energy by means of a RTICC. The strategy to overcome such challenge is based on a novel trilateral thermodynamic cycle. The proposed RTICC is not based on the quadrilateral Carnot cycle because the proposed thermal engine cycle absorbs energy and develops mechanical work simultaneously during the same process evolution or transformation. As consequence of this contribution this cycle is not restricted by the Carnot factor constraints. Its maximum thermal efficiency is influenced not only by the ratio of the top to the bottom temperature but also by some factors such as the structural characteristics of the proposed engine with regard to the regeneration capabilities. The proposed RTICC converts thermal energy to mechanical energy in a manner similar to the Otto or Diesel cycles by means of the known cylinderpiston structure.

\section{1. Analysis of the regenerative TTC}

Considering a regenerative cycle whose $\mathrm{T}$-s diagram is depicted in Fig. 1, follows that, the amount of specific energy added to the transformation (2)-(3) considering the transformation (2)-(3) as an open system is defined by equation (2).

Thus, the amount of heat that can be regenerated is defined as

$q_{R}=\left(h_{3}-h_{3 x}\right)$

$$
\begin{aligned}
& q_{i}=q_{23}-q_{R}=\Delta h_{23}+W_{23}-\left(h_{3}-h_{3 x}\right)= \\
& W_{23}-h_{2}+h_{3 x}
\end{aligned}
$$

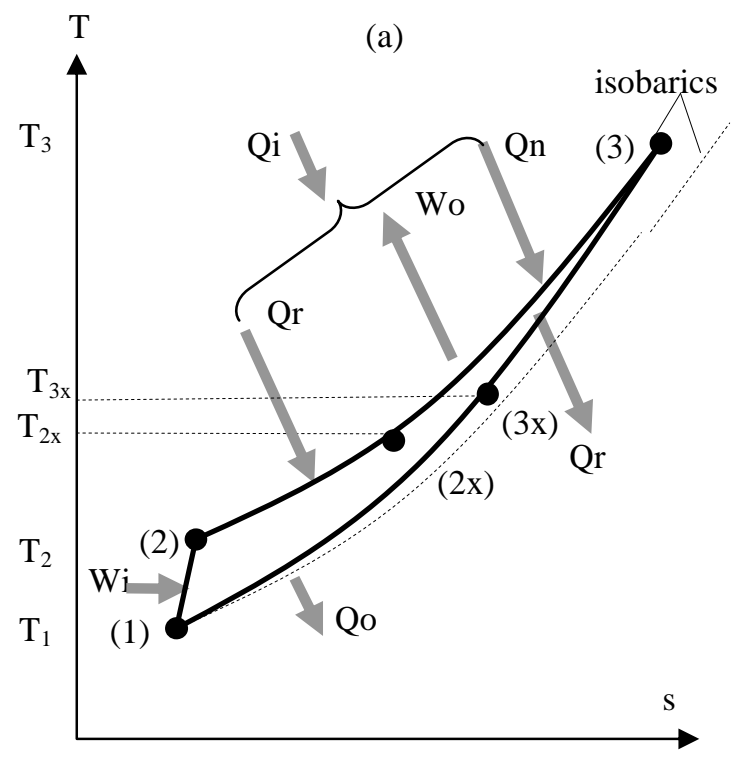

Fig. 1. The T-s diagram the RTICC.

The amount of heat rejected to the low temperature sink is $q_{o}=\left(h_{3 x}-h_{1}\right)$

hence the amount of thermal energy converted into work is the net work $W_{N}$ defined as

$W_{N}=q_{i}-q_{o}=W_{23}-W_{12}=W_{23}-h_{2}+h_{1}$

and the thermal efficiency is given as

$\eta_{R}=\frac{W_{N}}{q_{i}}=\frac{q_{i}-q_{o}}{q_{i}}=\frac{W_{23}-h_{2}+h_{1}}{W_{23}-h_{2}+h_{3 x}}$

According to the expression (5) the thermal efficiency for a regenerative TTC increases with the decreasing of the temperature $T_{3 x}$, so that the maximum thermal efficiency will be achieved when the temperature $T_{3 x}$.equals the temperature $T_{2}$, which will means considering the use of a reversible regenerator.

According to the T-s diagram shown in Fig. 1, in order to satisfy the 2nd law of the thermodynamic, after irreversible losses are neglected, the following conditions must be fulfilled.

$2^{\text {nd }}$ law fulfilment:

$T_{3 x} \geq T_{2}$

$T_{3} \geq T_{2 x}$

$T_{3} \geq T_{3 x}$

$T_{2 x} \geq T_{2}$

In an endeavour to find the maximum achievable thermal efficiency of the studied cycle further analysis is required. The following considerations must be taken into account, that means rejecting the minimum amount of heat $Q o$, and/or maximum net work delivered $W n$ for a given net input rate of heat flow, which implies the maximization of the expression (10) or the minimization of the expression (11), both expressed as

$\operatorname{Max}(W n) \rightarrow \operatorname{Max}\left[W o-\left(h_{2}-h_{1}\right)\right]$ 
$\operatorname{Min}(Q n) \rightarrow \operatorname{Min}\left[\left(W o+h_{3 x}-h_{2}\right)\right]$

In agreement with the information provided by Fig. 1, the cycle must satisfy the condition $\left(T_{3 x} \geq T_{2}\right)$. Furthermore, requirements for practical realization demand a temperature difference $\left(T_{3 x}-T_{2}\right)$ of at least 10 degrees $\mathrm{K}$ in order to allow an acceptable heat transfer flow. As consequence of such restriction, a compromise must exist between the aforementioned temperature difference and the heat transfer flow which definitely affects the size of the regenerator as well as some components of the plant.

\section{Case study: The proposed internal combustion engine}

A prototyping development task has been carried out based on the proposed case study starting from a preliminary design study and prototyping phase. This case study is referred to the cycle model described by equations (1-5), according to the structure shown in Fig. 2 under single (Fig. 2(a) or double effect (Fig. 2(b). The main purpose is to show that the proposed regenerative trilateral thermal cycle under a Otto-like physical structure can be arranged to render mechanical power at effective cost in comparison with conventional quadrilateral Carnot dependent thermal cycles such Otto and Diesel cycles.

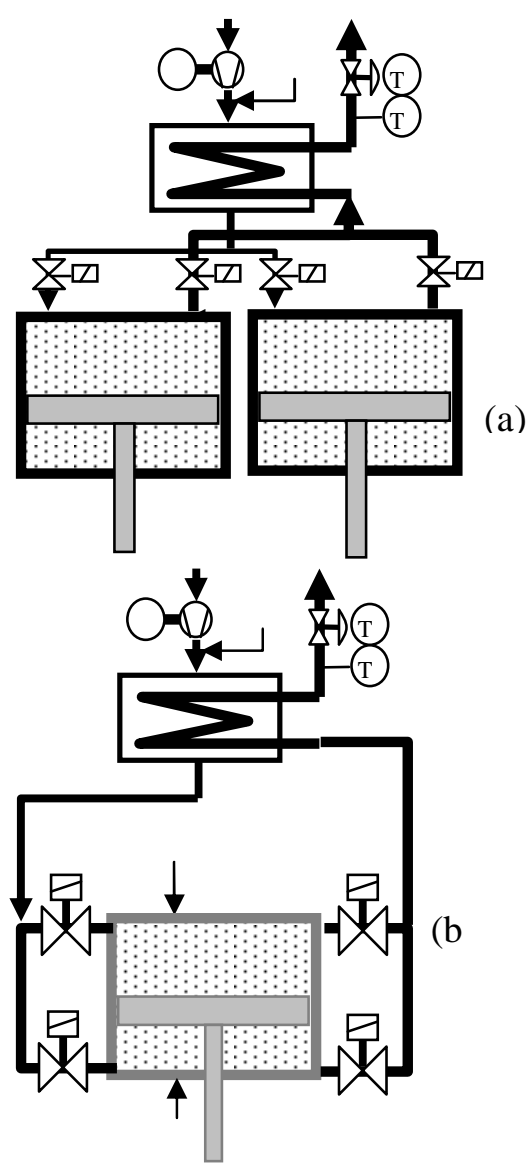

Fig.2. Schematic diagram of the projected RTICC based engine. (a), single effect. (b), double effect.
While air enters the cylinder during an outward stroke, the ignition of the air-fuel mixture is responsible for the power stroke carried out during such outward stroke (2-3). When the piston reaches the end of the power stroke (3), the backwards movement of the cylinder starts draining the hot flue gas through a heat regenerator which transfers the high grade heat from the exhausted gases to the combustion air previously compressed (31). Fig.1.

\subsection{Structure of the reciprocating internal combustion} engine based on a trilateral thermal cycle

Each piston movement (outward or inward stroke) is interpreted as a stroke of the trilateral regenerative internal combustion cycle (RTICC). The RTICC consists of a compression phase carried out by an external compressor that transfers air from the atmosphere to one of the cylinders, corresponding with the cycle process (1-2) carried out between the state points of the T-s diagram shown in Fig. 1.

\subsection{The heat source}

The proposed cycle RTICC is not based on a constant pressure heat addition cycle during a fraction of the stroke, which is often referred to as the Diesel cycle, but to another special case of an internal combustion engine, whose combustion is controlled so that the heat is absorbed during a complete expansion stroke which occurs at a pressure such that the generated force on the piston compensates the reaction load in the opposite side of the piston. This means an isobaric expansion (constant pressure) at constant load. Since the load may in general be variable, then the pressure is also variable as function of the load on the piston.

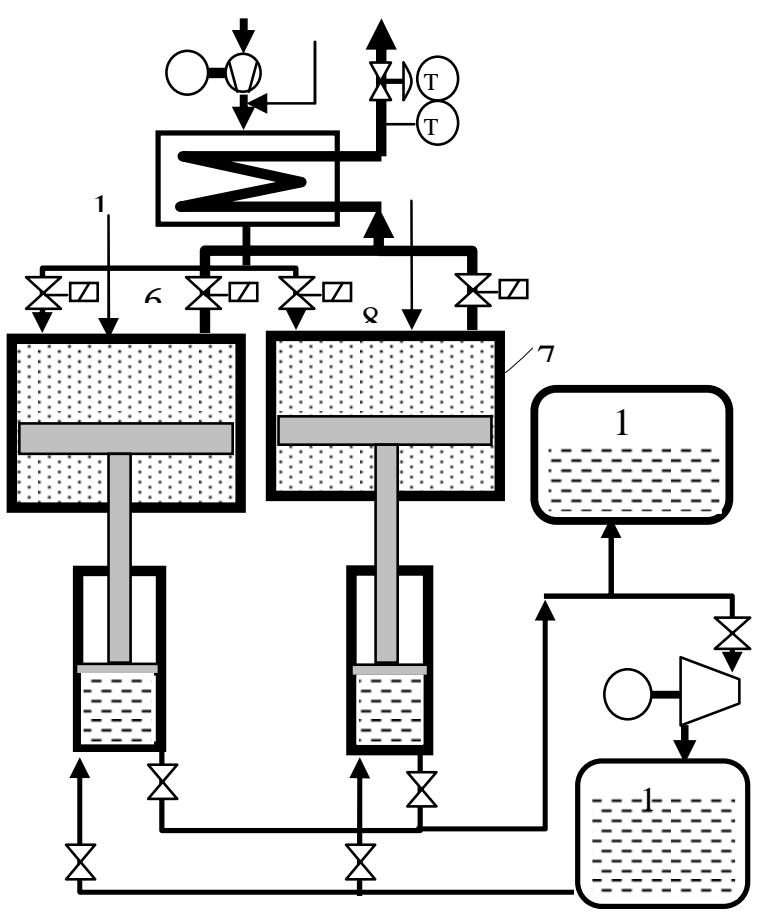

Fig. 3. RTICC engine structure with mechanical conversion by hydraulic techniques.

As shown in Fig. 2 and Fig 3, the combustion air is heated as much as possible by regeneration of the heat energy of flue gases which are exhausted at high grade heat value, 
i.e. at high temperature so that exhausted heat is used to heat the combustion air. This means that as the combustion air is being heated into the regenerator the flue gases are being cooled accordingly. The objective is to reject the minimum possible amount of heat carried with the exhausted gases to the heat sink (the environment).

Pure $\mathrm{CH}_{4}$ has been assumed for the theoretical preliminary design study. Thus, in Table 1 the combustion components considered for the case study are shown.

Table 1. Combustion byproducts expressed with respect to the air mass flow rate with $\mathrm{AFR}=194.8$

\begin{tabular}{|c|c|c|c|c|c|}
\hline \multicolumn{2}{|c|}{$\begin{array}{l}\text { Combstion } \\
\text { fuel/oxidant }\end{array}$} & \multicolumn{4}{|c|}{ Combustion Byproducts } \\
\hline Air & $\mathrm{CH}_{4}$ & $\mathrm{CO}_{2}$ & $\mathrm{H}_{2} \mathrm{O}$ & $\mathrm{N}_{2}$ & $\mathrm{O}_{2}$ \\
\hline$[\mathrm{kg} / \mathrm{s}]$ & {$[\mathrm{kg} / \mathrm{s}]$} & {$[\%]$} & {$[\%]$} & {$[\%]$} & {$[\%]$} \\
\hline 1 & $9.364 \cdot 10^{-3}$ & 1.4 & 1.15 & 76.58 & 20.87 \\
\hline
\end{tabular}

The AFR has been chosen so that a mean temperature of $760 \mathrm{~K}$ for the flue gases is achieved. Furthermore, flue gas temperature is set to the value of $760 \mathrm{~K}$ in order to be supported by the combustion chamber materials without damaging risks.

\section{The thermal cycle performance}

In the proposed case study the engine structure shown in Fig. 2 is considered. Depending on the top temperature, an appropriate air-fuel ratio will be chosen. As it will be highlighted from the analysis of the cycle and results, this cycle renders higher efficiency than the conventional Otto or Diesel indeed at low temperatures which means that a lower combustion temperature means to reduce nitrogen oxides.

In Table 2 the data corresponding to the analysed cycle is presented. This cycle is referred to the T-s diagram of Fig 1. The cycle performance is achieved by applying equation (5)

Table 2. Thermodynamic data for cycle analysis with data from [7]

\begin{tabular}{|l|l|l|l|l|l|}
\hline & $\begin{array}{l}\text { p_i } \\
{[\mathrm{kPa}]}\end{array}$ & $\begin{array}{l}\text { T_i } \\
{[\mathrm{K}]}\end{array}$ & $\begin{array}{l}\mathrm{h} \_\mathrm{i} \\
{[\mathrm{kJ} / \mathrm{kg}]}\end{array}$ & $\begin{array}{l}\text { s_i } \\
{[\mathrm{kJ} / \mathrm{kg} \cdot \mathrm{K}]}\end{array}$ & $\begin{array}{l}\mathrm{v} \_\mathrm{i} \\
{\left[\mathrm{m}^{3} / \mathrm{kg}\right]}\end{array}$ \\
\hline 1 & 101.3 & 288 & 288.4 & 5.661 & 0.816 \\
\hline 2 & 120.0 & 303.2 & 303.6 & 5.664 & 0.7252 \\
\hline $2 \mathrm{x}$ & 120.0 & 537.5 & 542.1 & 6.245 & 1.286 \\
\hline 3 & 120.0 & 760 & 778.5 & 6.613 & 1.818 \\
\hline $3 \mathrm{x}$ & & 353.2 & 33.9 & & \\
\hline
\end{tabular}

It must be considered that the combustion at a given air/fuel ratio allows us to choose the maximum temperature according to data extracted from [7] as well as the tool Engineering Equation Solver (EES) which are relevant tools that contribute to a substantial improvement of the cycle's analysis capabilities.

\subsection{Discussion of results}

Although a set of relevant results of the RTICC are presented, this preliminary design study has been carried out on the basis of theoretical considerations, by neglecting the losses inherent to any real engine. To approximate the results of the study to a real plant, it will be necessary to take into account the inherent losses due to internal irreversibilities, which certainly involve losses mainly due to heat transfer to the surroundings, isentropic efficiency of the air blower, working fluid friction and mechanical losses. However, the theoretical achieved results give us a useful guide to continue on the development of a real efficient engine.

Consequently, as result of computed cycle data shown in Table 2 with a working fluid described in Table 1, the achieved results are discussed. Thus, in Table 3 the thermal efficiency and delivered specific work are presented as function of operating pressures at maximum operating temperature of $\mathrm{T}_{3}=760 \mathrm{~K}$, and Carnot Factor $\mathrm{CF}=62.11(\%)$. The achieved data shows that as pressure increases from 120 to $500 \mathrm{kPa}$, the thermal efficiency decreases from $44.66 \%$ to $8.95 \%$. Furthermore, for operating pressures lower than $200 \mathrm{kPa}$, the thermal efficiency is lower than the Carnot factor. With respect to the delivered specific work, follows that as pressure increases from $120 \mathrm{kPa}$ to $500 \mathrm{kPa}$, the specific work decreases also from $204.9 \mathrm{~kJ} / \mathrm{kg}$ to $41.07 \mathrm{~kJ} / \mathrm{kg}$. These unexpected results suggest us that to achieve better performance (acceptable thermal efficiency and specific work), lower operating working pressures are required. However, as operating pressures decreases the specific volume increases almost proportionally so that a compromise between the engine (mass and volume) and the thermal efficiency associated with the specific work must be established. These results are graphically depicted in Fig. 4.

Table 3. Thermal efficiency and specific work as function of the operating pressure for $\mathrm{T}_{3}=760(\mathrm{~K})$, and $\mathrm{CF}=62.11$ $(\%)$

\begin{tabular}{|l|l|l|l|}
\hline $\mathrm{p}_{3}[\mathrm{kPa}]$ & $\eta(\%)$ & $\mathrm{Wn}[\mathrm{kJ} / \mathrm{kg}]$ & $\mathrm{v}_{3}\left[\mathrm{~m}^{3} / \mathrm{kg}\right]$ \\
\hline 120 & 44.66 & 204.9 & 1.835 \\
\hline 140 & 41.49 & 190.4 & 1.573 \\
\hline 160 & 38.62 & 177.2 & 1.376 \\
\hline 180 & 36.00 & 165.2 & 1.223 \\
\hline 200 & 33.57 & 154.0 & 1.101 \\
\hline 300 & 23.51 & 107.9 & 0.734 \\
\hline 400 & 15.59 & 71.52 & 0.551 \\
\hline 500 & 8.95 & 41.07 & 0.440 \\
\hline
\end{tabular}

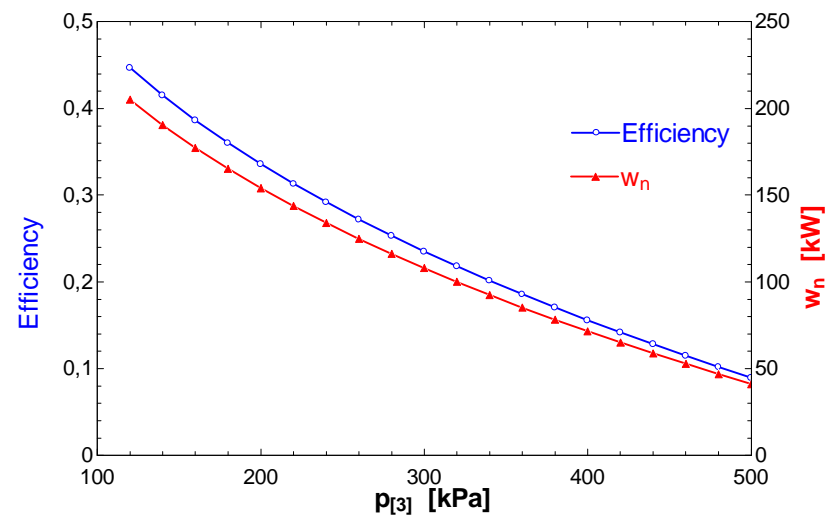

Fig. 4. Thermal efficiency and specific work as function of the operating pressure for $\mathrm{T}_{3}=760(\mathrm{~K})$, and $\mathrm{CF}=62.11$ $(\%)$. 
With respect to toe possible operating temperatures, according to the data shown in Table 4, as the top operating temperatures $\mathrm{T}_{3}$ varies from $500 \mathrm{~K}$ to $1000 \mathrm{~K}$ at a top pressure of $\mathrm{p}_{2}=120 \mathrm{kPa}$, follows that the thermal efficiency increases slightly from $34.38 \%$ with $\mathrm{CF}=71.0$ $\%$ to $66.13 \%$ with $\mathrm{CF}=52.0 \%$, and the specific work increases from $276.5 \mathrm{~kJ} / \mathrm{kg}$ to $157.8 \mathrm{~kJ} / \mathrm{kg}$. The corresponding graphic results are depicted in Fig. 5.

Table 4. Thermal efficiency, specific work and Carnot factor as function of the operating temperatures for $\mathrm{p}_{2}=\mathrm{p}_{3}$ $=120 \mathrm{kPa}$.

\begin{tabular}{|l|l|l|l|}
\hline $\mathrm{T} 3[\mathrm{~K}]$ & $\eta(\%)$ & $\mathrm{Wn}[\mathrm{kJ} / \mathrm{kg}]$ & $\mathrm{CF}(\%)$ \\
\hline 600 & 66.13 & 157.8 & 0.52 \\
\hline 700 & 49.88 & 187.2 & 0.59 \\
\hline 800 & 42.08 & 216.8 & 0.64 \\
\hline 900 & 37.46 & 246.5 & 0.68 \\
\hline 1000 & 34.38 & 276.5 & 0.71 \\
\hline
\end{tabular}

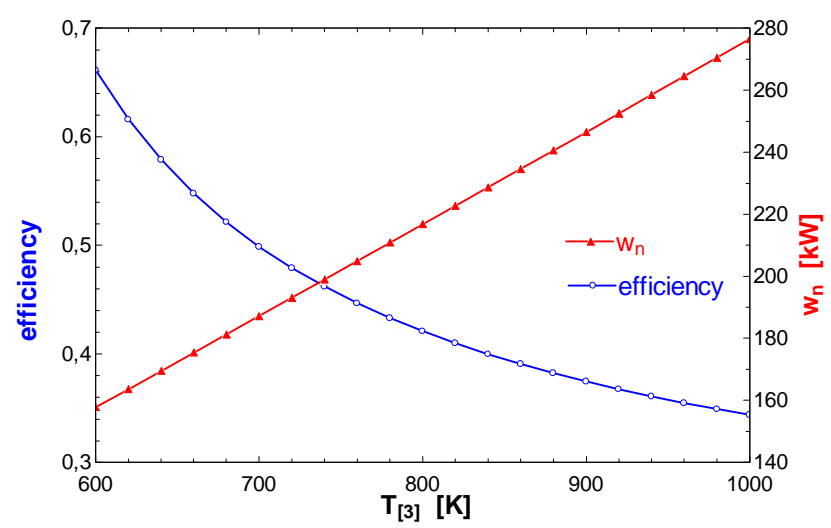

Fig.5. Thermal efficiency, specific work and Carnot factor as function of the operating temperatures for $\mathrm{p}_{2}=\mathrm{p}_{3}=120$ $\mathrm{kPa}$.

In most of the engines operating with conventional thermal cycles, the bottom temperature is important due to its influence on the thermal efficiency and specific work. However in the proposed cycle, as shown in Table 6, the ambient temperature hasn't significant influence on the thermal efficiency and specific work at such low operating pressure $(120 \mathrm{kPa})$.

Table 6. Thermal efficiency, specific work and Carnot factor as function of the operating bottom temperatures (environment) with $\mathrm{T}_{3}=760 \mathrm{~K}$ and $\mathrm{p}_{2}=\mathrm{p}_{3}=120 \mathrm{kPa}$.

\begin{tabular}{|l|l|l|l|}
\hline Tref $[\mathrm{k}]$ & $\eta(\%)$ & $\mathrm{Wn}[\mathrm{kW}]$ & $\mathrm{CF}(\%)$ \\
\hline 273 & 44.84 & 205.7 & 64.08 \\
\hline 278 & 44.78 & 205.4 & 63.42 \\
\hline 283 & 44.72 & 205.2 & 62.76 \\
\hline 288 & 44.66 & 204.9 & 62.11 \\
\hline 293 & 44.60 & 204.7 & 61.45 \\
\hline 298 & 44.55 & 204.8 & 60.79 \\
\hline 303 & 44.49 & 204.1 & 60.13 \\
\hline 308 & 44.43 & 203.9 & 59.47 \\
\hline
\end{tabular}

According to this preliminary analysis, the thermal engine operating under a RTICC exhibits as a viable thermal cycle in which deep experimental studies are required to verify its real performance. It appears to be reasonable accepting that assuming the difference of ideal thermal efficiency with respect to Carnot engine, an approach to the same difference will be expected with respect to a real thermal engine.

\section{Conclusions}

A novel regenerative trilateral thermal cycle characterized by relevant differences with respect to conventional quadrilateral thermodynamic cycles has been proposed. As stated before such differences deal with the tasks of absorption and conversion of energy into mechanical work, which are performed simultaneously during a single transformation.

The most important conclusion is related to the achieved thermal efficiency of the cycle which exceeds the Carnot efficiency for all studied combinations of pressures temperatures and air-fuel ratios. The main reasons for the efficiency improving with respect to conventional power cycles is due to the association of the following contributions which depends on the plant structure:

- The cycle absorbs heat and simultaneously converts it into mechanical work in a single cycle transformation.

- Inherent regeneration.

- The chosen operating parameters (mainly chosen working pressures).

Finally the widespread use of residual heat as power sources as well as the fact of increasing the thermal efficiency apart from the fact of contributing to reducing the massive use of fossil fuels is strongly associated with the reduction of ozone depletion potential and the global warming potential.

\section{References}

[1] Johann Fischer, Comparison of trilateral cycles and organic Rankine cycles. Energy 2011; 36: 6208-6219, http://dx.doi.10.1016/j.energy.2011.07.041;

[2] Yingru Zhao, Jincan Chen, An irreversible heat engine model including three typical thermodynamic cycles and their optimum performance analysis. International Journal of Thermal Sciences 2007; 46: 605-613.

[3] Yanlin Ge, Lingen Chen, Fengrui Sun, Chih Wu, Thermodynamic simulation of performance of an Otto cycle with heat transfer and variable specific heats of working fluid. International Journal of Thermal Sciences 2005; 44: 506-511

[4] Shaojun Xia, Lingen Chen, Fengrui Sun, Engine performance improved by controlling piston motion: Linear phenomenological law system Diesel cycle. International Journal of Thermal Sciences 2012; 51: 163174

[5] S.C. Kaushik, V.SivaRedd, S.K.Tyagi, Energy and exergy analysis of thermal power plants: A review. Renewable and Sustainable Energy Reviews 2011; 15: 1857-1872.

[6] Jing Li, Gang Pei, Yunzhu Li, Dongyue Wang, Jie Ji, Energetic and exergetic investigation of an organic Rankine cycle at different heat source temperatures. Energy 2012; 38: 85-95. 
[7] E. W. Lemmon, M. L. Huber and M. O. McLinden,. NIST Reference Fluid Thermodynamic and Transport Properties - REFPROP Version 8.0, User's Guide, NIST 2007, Boulder, Colorado. 